\title{
Device and Method of Formation of Porous Nutritional Coating on Seeds
}

\author{
Ermat Kubeev ${ }^{1, *}$ and Viktor Smelilk ${ }^{2}$ \\ ${ }^{1}$ Federal State Budgetary Educational Institution of Higher Education «Yaroslavl State Technical \\ University» (YSTU), Yaroslavl, Russia \\ ${ }^{2}$ Federal State Budgetary Educational Institution of Higher Education «Saint-Petersburg State \\ Agrarian University» ( FSBEI HE SPb SAU), Saint Petersburg, Russia.
}

\begin{abstract}
Modern technologies for applying artificial shells are based on the principles of rolling or granulating with subsequent drying. This technology does not allow to get a gas - permeable porous shell, which often leads to the death of seeds during the formation of the shell, storage and transportation, and slow growth at the early stages of plant development. The aim of the research is to develop a new method and device for pre-sowing treatment of agricultural seeds, which can increase their germination, germination energy and ensure crop growth. A distinctive feature of the new method is that the shell is formed not by rolling, but by molding around the seed, followed by drying in a soft mode. This will form a porous, gas-permeable structure around the seed. Based on the mathematical description of the processes occurring during the introduction of seeds into the nutrient medium, the design calculations and calculations made a laboratory stand for the study of the seeds coating with a protective and nutritious shell, which consists of a mixing, sealing and seeding nodes. A series of experiments was conducted to study the operation of the stand in various modes. Optimal operating modes are identified.
\end{abstract}

\section{Introduction}

One of the effective methods for pre-sowing preparation of seeds is their pelleting - coating the seeds with a special mixture. It creates a protective and nourishing membrane, increasing their size and giving the seeds the right shape. This allows us to ensure normal seed nutrition in the early stages of development, when the root system of the plant is still weak or not formed. This is especially effective when the soil contains a small amount of elements necessary for plant development (Kubeev, E,2015). Despite the obvious advantages of this method, its development is constrained by a number of reasons, the main of which is the application technology, based on the use of rolling principles or granulation with subsequent drying. With such methods, the resulting shell has low gas and moisture permeability, which often leads to seed death during shell formation, storage and transportation. In addition, there is slow growth in the early stages of plant development.

\footnotetext{
* Corresponding author: kubeevei@mail.ru
} 
Having analyzed the current state of the of applying shells problem to seeds, it was found that a fairly large number of scientists in different countries of the world are involved in these studies (Kubeev, E, 2015 - Lebedev, 2015). Despite the variety of coating methods, types and materials of coating compositions, application modes, the rolling method is almost always applied in which the above problems arise.

In addition to the classical rolling methods, some researchers are experimenting with the properties of forming composition materials. In order to improve the quality of granular seeds, the authors of (Woomer, 2010) propose a method of applying a shell formed from a suspension and a binder material. However, this method, like many others does not solve the problem of gas permeability of the protective and nutritional shell. There is also a number of works on the influence of the structure surrounding composition on gas and moisture permeability. Researchers in (The effect of parsley seed hydration treatment and pelleting on seed vigour Plant soil environ.,2003) present the results of the small-seeded crops respiratory activity study.

Therefore, the problem of formation of gas- and moisture-permeable protective nutritional coating during pre-sowing treatment of seeds (PSTS) remains a live issue and has not been fully solved.

\section{Material and methods}

After analyzing the existing shortcomings of granular seeds and the methods of forming protective-nutritional shells, the authors of the work proposed a method of applying nutritional compositions that provides protection and supply of seeds with the necessary substances in the early stages of development. A distinctive feature of this method is that the shell is formed not by rolling, but by molding around the seed, followed by drying in a soft mode. This will allow the formation of a porous, gas-permeable structure around the seed, with a size of not more than 3 seed diameters.

In our case, a porous gas-permeable shell (PGS) will not prevent the penetration of moisture and air to the seed, which will prevent its death and reduce the cost of watering. The application of a porous shell in spherical cells with subsequent compaction is assumed. According to our method, spherical (or other shape depending on the shape of the seeds) cells of high porosity are formed from the prepared tearing composition, which are the lower and upper halves of the future shell. Grain is placed in the lower cell, which is then closed by the upper part of the shell, followed by a slight compaction, necessary for the formation of a single spherical (or other shape) granule.

Volume of seeds and components in a two-cell capsule (Figure 1) [2]:

$$
V_{c}=2 \beta f_{c} l
$$

where $\beta$ is the filling factor of cells (for small-seed vegetable crops $\beta=0.9$ ); $f_{c}$ is the sectional area of cells; $l$ is the drum length.

With the selected $d$ diameter of the drum, the volume of seeds and pelleting components to be placed in a two-cell capsule is determined as follows:

$$
\begin{gathered}
V_{c}=2\left(V_{1}+V_{2}\right) \\
V_{1}=\frac{d^{2}}{4} l(\alpha-\sin \alpha)
\end{gathered}
$$

Calculation of $V_{2}$ determine the following: 

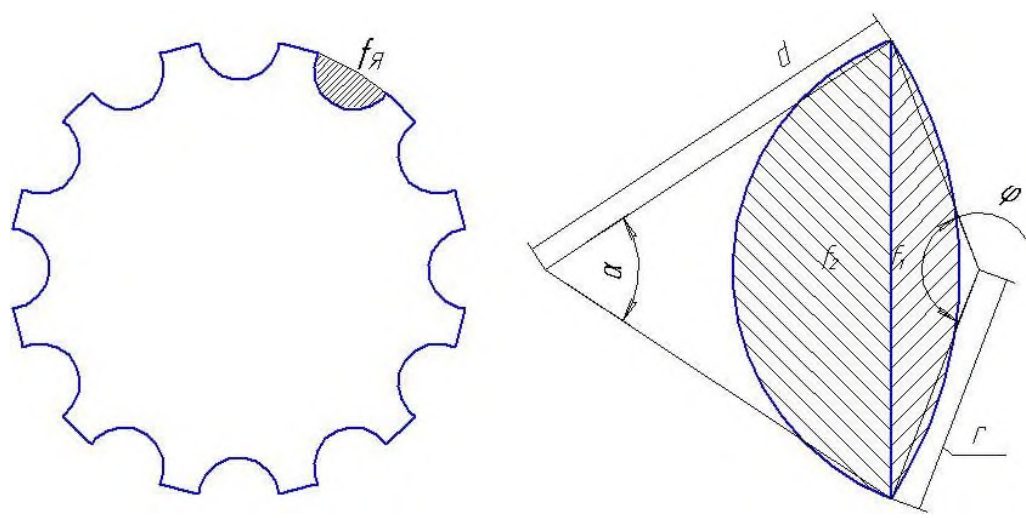

Fig. 1. Construction of pelleting drum and its cell

$$
V_{2}=\pi r^{2} l
$$

During the formation of the shell, air is released in the material. In this case, numerous channels are formed, as a result of which the resulting shell has a porous structure, easily absorbs moisture and does not prevent air from entering the seed.

The application of the proposed method of coating seeds PGS will increase their germination and provide early sowing. The presence of pesticides in the shell protects the plant from pests. In the production of pelleted seeds by the proposed method, a mixer, a device for compaction (shell formation) and a sowing apparatus are necessary. To implement the proposed PSTS method, it is also necessary to study the process of precise sowing of seeds of coarse crops (CLCA), the seeds of which have an irregular shape.

The new method is supposed to be carried out directly when sowing seeds in the soil using an aggregate mounted on a vehicle. The unit should include a compaction device, consisting of rollers for forming cells in the soil and compaction, and a metering unit. First, using a mixing device, a mixture of the components of the protective and nutritional composition is prepared. Next, the prepared mixture is loaded into the hopper with a dispenser and, when the unit is moving, enters the cells made using the compaction device. Then, in each partially filled cell with the help of a sowing device, one seed is fed, filled with a protective-nutrient mixture and compacted. On top of the resulting granule is covered with soil. One of the most important stages in the formation of granules is the process of mixing the components of the coating composition. The main problem of preparing a mixture of shell components is that its constituent components are very different in particle size and weight content. In order to increase the homogeneity of the mixture, we proposed first to mix mineral fertilizers, microelements and finely divided filler, and then to introduce the finely divided dry peat composition into the resulting mixture in the second phase. As a mixing device, it is most effective to use a brush apparatus (Figure 2). Liquid binders are added to the mixture by spraying. Preliminarily, the preparation of finely dispersed lowland peat was carried out in stages: drying, grinding, sieving. 


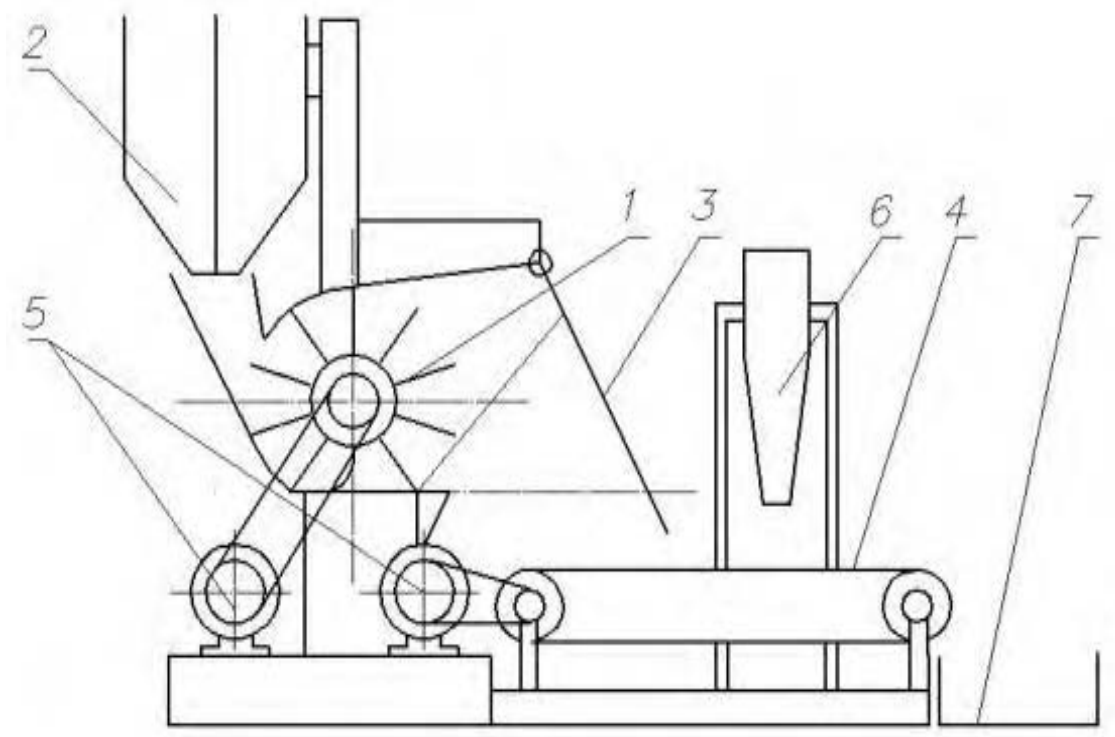

1-brush drum, 2-feeders of bulk components, 3-chipper, 4-conveyor, 5-drive, 6-sprayer of liquid binders, 7-container for collecting the finished product

Fig. 2. Mixing unit Diagram
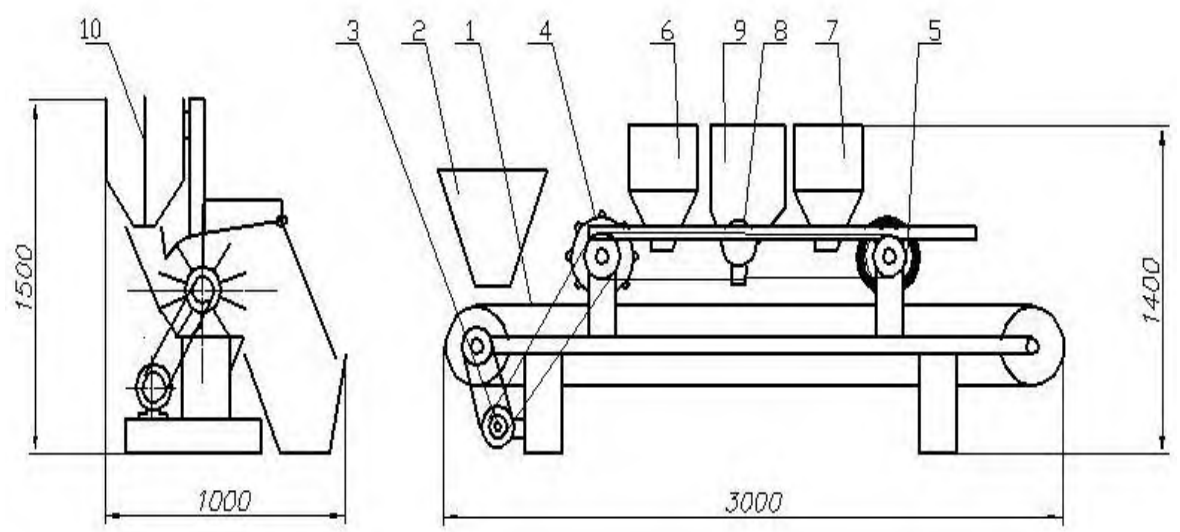

Fig. 3. Diagram of the device

Figure 3 shows a diagram of the device. When the device is operating, first with the help of a hopper 2 with a dispenser, a soil layer 30-50 mm thick is formed with a thin layer on the tape in the lower part of the tape (imitation of real field conditions). Further, when passing the roller 4 in the layer, cells having the shape of a hemisphere are pressed. Then, from the hopper 6 , with the help of a batch type dispenser, a pre-prepared mixture of nutrients. It consists of fine sifted peat, microelements, mineral fertilizers, liquid manure, which simultaneously serves as a binder. And it will laiding in the cells. A mixer 10 is used to prepare the mixture. Bulk components are mixed separately. 
In each filled cell from the sowing apparatus 8, one seed is placed. Then, a similar mixture from the hopper 7 with a dispenser is introduced into the cells with seeds. Using the roller 5, having on the surface a shell of elastic material, the nutrient mixture in the cell is compacted. At the same time, a granule is formed around the seed from a material of nutritional composition.

\section{Results and discussion}

In order to identify rational operating modes of the mixing unit, experimental studies were conducted. Bulk materials to be mixed (peat, trace elements, mineral fertilizers) in appropriate quantities were poured into each of the bunker compartments. When the unit was turned on, by adjusting the current supplied to the DC motors, the speed of the spray drum was adjusted. Then the hopper flaps were opened, and for different ratios of material consumption, the flaps were opened at different distances. Particles of materials fell into the working area of the spray unit, where they were captured by rotating elastic elements, and were brought into complex motion with the formation of a rarefied conical torch. At the same time, having significant speed and different directions of motion, the components were mixed in a torch and hit the receiver (in the presence of a fencing element, particles of the reflected stream fell into the receiver). The operation of the installation was stopped when the entire contents of the bunkers were completely poured out. Samples were taken from the receiver, and the heterogeneity coefficient of the resulting mixture was found by known methods.

In the experiments, the following values varied: drum rotation frequency and beat deformation. One of the main parameters that affect the value of the heterogeneity coefficient is the angle of the baffle element inclination. This dependence is shown in Figure 4 for a two-component mixture of sifted peat with mineral fertilizers. The dots on the graph represent the experimental data, the solid line represents the regression dependencies (Lebedev, 2015). It was found that with an increase in the angle of the chipper inclination, the quality of the mixture increases, which is obviously explained by an increase in the interaction zone of the reflected and incident flows in the working volume of the apparatus and, therefore, an increase in the number of particles collisions in the flows.

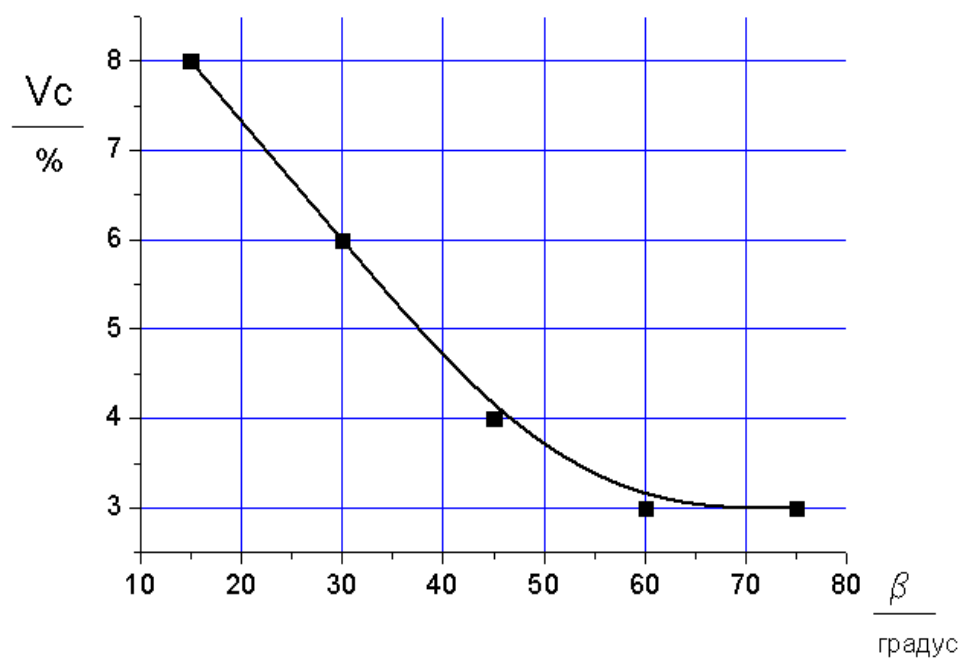

Fig. 4. Dependence of the mixture inhomogeneity coefficient on the angle of inclination 


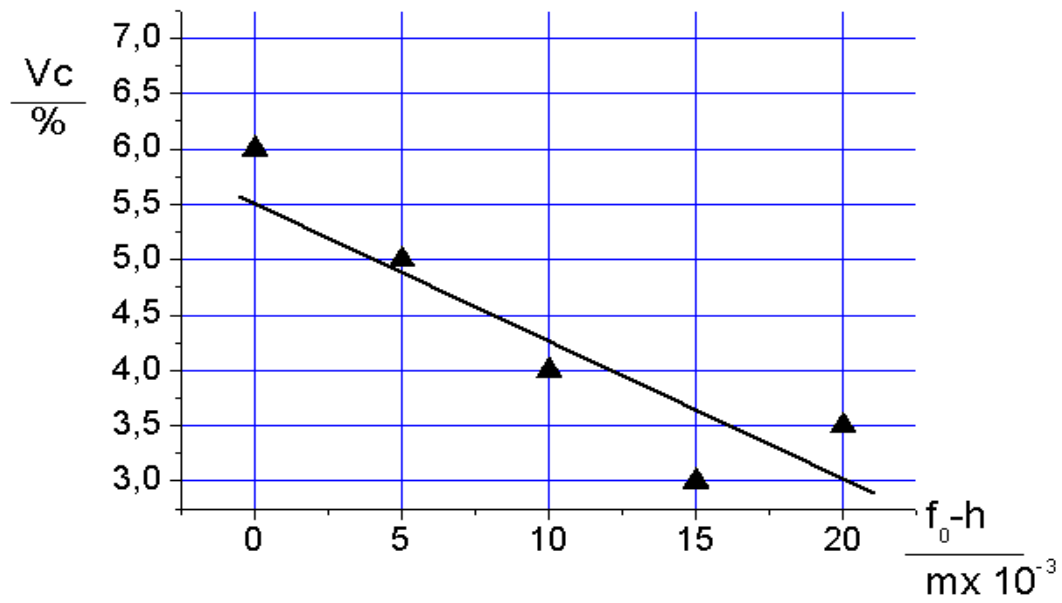

Fig. 5. Dependence of the mixture inhomogeneity coefficient on the bill deformation

In Figure 5 shows the dependence of the of the mixture heterogeneity coefficient on the deformation of flexible elements (beat). As expected, with an increase in the deformation of flexible beats, the quality of the mixture increases. Obviously, this is due to the same reasons, i.e. due to an increase in the randomization of the bulk materials volumes movement due to an increase in flow rates. It should be borne in mind that the effect of "crushing" of materials volumes into smaller ones moving together also arises. However, a further increase in deformation leads to an increase in the of mixture heterogeneity coefficient, which, apparently, is affected by an increase in friction against each other and on the surface of the tape.

The influence of the rotational speed on the mixture heterogeneity coefficient is shown in Figure 6. It can be seen from the graph that with an increase in the speed of the atomizer rotation, a decrease in the coefficient of heterogeneity is observed. With an increase in the particle velocity in the flow, an improvement in the quality of the mixture takes place. The latter is explained by the fact that at high speeds of particles, the randomness of their motion in the plume also increases. The increasing effect of air flow is also affecting.

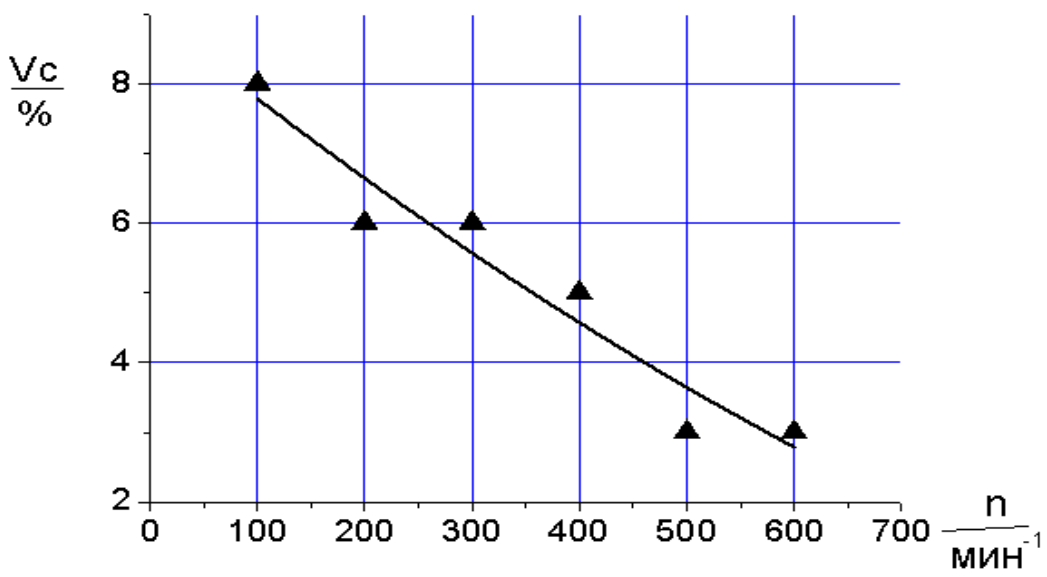

Fig. 6. The dependence of the mixture heterogeneity coefficient on the speed 
When conducting experiments on mixing materials, the following values of operating and design parameters were established at which the coefficient of heterogeneity of the mixture has a minimum value (not more than 6\%). Table 1 presents the recommended values of the rotational speed, the angle of the chipper inclination and the degree of the beater deformation when mixing materials of nutritional composition.

Table 1. Recommended values of operating parameters

\begin{tabular}{|l|c|c|c|}
\hline & $\mathrm{n}, \mathrm{min}-1$ & The degree of deformation & the angle of inclination \\
\hline Peat-mineral fertilizers & 700 & 1,4 & 750 \\
\hline $\begin{array}{l}\text { Peat-an inert filler } \\
\text { kaolin) }\end{array}$ & 750 & 1,6 & 700 \\
\hline $\begin{array}{l}\text { Trace elements - mineral } \\
\text { fertilizers }\end{array}$ & 700 & 1,5 & 800 \\
\hline
\end{tabular}

To assess the quality of the sowing apparatus, expressed in the uniformity of sowing seeds, it is necessary to trace the movement of seeds in all phases, as well as the final placement of seeds in the cells. Taking into account these requirements, a special device was developed and manufactured for conducting experimental research, which allows conducting research on the operation of the sowing unit. To fix the sown seeds, traps were attached to the movable tape, the surface of which is covered with glue. We studied the longitudinal and transverse uniformity of sowing, and also determined the pulsation of the sowing using a frame with marking lines. The uniformity of seed distribution was characterized by standard indicators of variation statistics with the determination of mean values, standard deviations and coefficients of variation.

Studies of the sowing apparatus were carried out in several stages. At the first stage, the quality of sowing corn seeds by the sowing apparatus was evaluated. The second studied the effect of the seeding rate on the uniformity of distribution. The seeding rate is determined by the rotation speed of the metering unit shaft and the speed of the conveyor belt. The limits of these indicators variation are as follows: seeding rate - from 5 to 20 pcs / $\mathrm{m}$, belt speed - from 0.01 to $0.06 \mathrm{~m} / \mathrm{s}$. The evaluation criteria for the quality of sowing were: longitudinal uniformity of seed distribution; transverse uniformity of seed distribution; pulsation of the sowing apparatus and the degree of damage to the seeds.

To determine the longitudinal and transverse uniformity of the seeds distribution, as well as the pulsation of the sowing apparatus, the seeds were sown on a paper tape coated with varnish. To simplify the analysis process, we used a partition of the entire width of the sown seeds into $\mathrm{n}$ sections with a subsequent analysis of the sowing density in each of them. For this purpose, a specially made marking frame with dimensions of $35 \times 15 \mathrm{~cm}$ was used, inside of which a grid with a mesh size of $10 \times 10 \mathrm{~mm}$ was woven.

As a result of the experiments, it was found that at the steady state of operation, the pneumatic sowing module allows the seeds to be placed in the prepared cells with the required accuracy, and the number of damaged corn seeds does not exceed $1 \%$. The process of forming a shell from a mixture prepared in the manner described above was studied on an experimental device and in the field. It was found that the granules thus formed have sufficient strength and have the porous structure necessary for air and moisture to enter the seed.

\section{Conclusion}

A new method has been developed for coating seeds of coarse-grained crops with a protective-nutritious coating. A series of experiments was carried out to study the operation 
of the installation in various modes. Rational operating modes are revealed. The developed method and device design for its implementation can be used at various agricultural enterprises from small farms to large agricultural firms. When applying the proposed method, it is expected to increase yields by $10-20 \%$ and reduce costs for expensive and time-consuming operations (thinning, pesticides and fertilizers) by $50 \%$.

\section{References}

1. E. I. Kubeev, Improving the efficiency of the technological process of pre-sowing seed treatment of agricultural crops by improving methods and technical means of applying artificial shells: dis. Dr. Tech. Sciences: 05.20.01, SPbSAU, 349 (2015)

2. A. I. Zaytsev, A. E. Lebedev, V. N. Sidorov, A. A. Murashov, A. V. Dubrovin, Pat. No.2388204 Russian Federation, IPC A 01/6. Device for coating of seeds of agricultural crops. Publ. 10/05/10, Bull. 13

3. A. I. Zaytsev, Pat. No. 2379871 Russian Federation, IPC A01C 1/06. Unit for applying coating compounds to seeds of agricultural crops - Publ. 01/27/10

4. V. A. Afanasev, Pat. No. 2733290 Russian Federation, IPC A01C 1/06. Pelleting Appatus, Publ. 10/01/20

5. I. V. Yudaev, Pat. No. Russian Federation, IPC A01C 1/06. Seed Pre-Sowing Treatment Device Publ. 07/27/20

6. Pol. L. Woomer, Seed Inoculation: Master Farmer Training Practical (2010)

7. Seed priming improves seed germination and reduces oxidative stress in black cumin (Nigella sativa) in presence of cadmium, 79, 195-204 (2016)

8. Quantification of seed-soil contact of sugar beet (Beta vulgaris) using X-ray Computed Tomography. Plant Methods, 13, 71 (2017)

9. Some physico-mechanical properties of carrot (Daucus carota L.), cherry belle (Rhaphanus sativus L.) and nigella (Nigella sativa L.) seeds coated with pellets Akademik Ziraat Dergisi, 8(1), 21-26 (2019)

10. Effect of Seed Pelleting on Seed Quality Parameters of Bell pepper (Capsicum annuum L.) under Laboratory and Nursery Conditions. International Journal of Microbiology Research, ISSN: 0975-5276 \& E-ISSN: 0975-9174, 11(1), 1452-1454 (2019)

11. V. A. Smelik, Presowing preparation of seeds by deposition artificial shells. SPbGAU $\mathrm{SPb}, 272$ (2011)

12. A. E. Lebedev, New apparatuses for processing dispersed materials in a rarefied state. Theory and calculation, 200 (Yaroslavl: Ed. YASTU, 2015)

13. E. I. Kubeev, The interaction of seeds and components dragee. Technique in agriculture, 3, 37-39 (2010)

14. E. I. Kubeev, Pat. No. 2437044 Russian Federation, IPC7 F26B 17/04. Dryer for bulk materials. Publ. 12/20/11

15. S. P. Timoshenko, O. M. Vechera, Seed mordants-comparative characteristics, problems and achievements $/ 3 \mathrm{~b}$. Ukrainian research Institute for forecasting and testing equipment. Agricultural machinery and technologies, 4, 9-14 (2010) 